\title{
Research on the Application of Planning Theory in the Material Delivery of EMUs Maintenance
}

\author{
Wang $\mathrm{Pu}$ \\ China Academy of Railway Sciences \\ Beijing, China \\ wp_sword@163.com
}

\author{
$\mathrm{Li} \mathrm{Li}$ \\ China Academy of Railway Sciences \\ Beijing, China \\ lili@163.com
}

\begin{abstract}
The paper focuses on various planning theories in Operations research. As the warehouses, workstation and marshalling yards are scattered across EMUs maintenance base with a long distance, and materials are not allowed to be stored in the workshops for a long time, under strict maintenance requirements in both time and quality, the paper offers the optimal solution for allocating staff and material using algorithm related to planning theories, catered to both car distribution and workstation distribution. Also, the RFID recognition technology will be used in distribution of material. This provides a supporting theory for efficient and detailed EMUs maintenance management.
\end{abstract}

Keywords-Operations Research, Linear Programming, Electric Multiple Units (EMUs) Maintenance, Car Distribution, Workstation Distributio

\section{INTRODUCTION}

Operations Research is an interdisciplinary study across math and formal science, using statistics, mathematical models and algorithms to find optimal or near optimal solutions for problems. It is often used to solve complicated issues in real life, especially in improving system efficiencies [1].

Planning theory, as a branch of Operations Research, focuses on how to use limited human, material, financial resources and time in the mathematically most efficient way to better accomplish the goal. Planning theory includes linear planning, non-linear planning, integer planning, goal planning and dynamic planning, etc [2]. The problem planning theory solves is that given certain circumstances, how to reach the extreme of object functions according to a certain parameter [3]. Specifically, linear planning could be used to solve material and staff allocation and delivery issues in Railway industry, while dynamic planning theory offers solutions to best optimal path, resource allocation, production coordination, stock control and equipment update issues [4].

Materials can be active and timely distribution in place by using linear programming theory combined with the EMU maintenance rules. This can speed up the material flow speed and improve maintenance efficiency. The range of delivery includes repair workstations, work station and operation depots in EMUs bases. Several distribution sections in EMUs maintenance base are from the distribution center to the repair workshop, from the distribution center to the work station, from the repair workshop to the work station, from the repair workshop to the warehouse, from the work station to the warehouse, etc.

\section{THE THEORY OF LINEAR PROGRAMMING}

Linear programming is put forward by the French mathematician Fourier in 1832 at the earliest. Linear programming is an important branch of operational research. It is to be studied earlier, and it is to be developed rapidly and widely. It is a mathematical method as to assist management by using in scientific means. It is focus on mathematical theories and methods of extremum problems of linear objective function under the linear constraint condition.

Generally, three steps is must be abided by when you build the mathematics model from the actual problem by using this method. The decision variables should be found according to the influence factors to achieve the purpose. The objective function is determined by the function relationship between decision variables and purpose. The restrictive conditions of the decision variables will determine how to satisfy the constraint conditions. When we get the objective function of the mathematical model is linear function, and constraints are linear equation or not, we call this mathematical model for the linear programming model.

\section{CURRENT METHOD FOR MATERIAL DELIVERY IN EMUS MAINTENANCE}

EMUs maintenance base is our country's important project in modernization of railway technical equipment, which is mainly responsible for EMUs comprehensive to repair tasks and manufacturing capacity. The EMUs of the maintenance base uses the production line and fault state as the main maintenance mode. The implementation of information technology in management and production is the basic guarantee of the production. The informatization of internal logistics distribution system is extremely important.

The logistics distribution of EMUs maintenance includes material requirements planning and material delivery- receipt etc. EMU maintenance parts are divided into two categories. A kind of parts called repair parts (RP) must be replaced. And the others called fault parts (FP) will be replaced if it is found to be fault. The RPs are used for advanced maintenance. Generally, they need to be delivered to a fixed station on time. The FPs are used when a fault is discovered during the maintenance process. They are packaged into a unit According to the experience, and then they are delivered to marshalling yards. Firstly, it is must to create a Demand planning, according to the requirement of the maintenance plan and 
tasks and fault processing, also combined with the technology of position requirements. When demand planning is approval, a BOM list will be created. After distributed in place, the parts in BOM list are received by material department. The maintenance will be continued. Then the inspector will check the quality of parts in the maintenance site, and the Accounting staff will make the consumption statistical sheet of parts.

\section{Designing THE APPLICATION}

At the moment, there is a distribution center for maintenance material in every EMUs base. Material for maintenance is storaged on the designated area. The delivery of all materials in EMUs bases is managed by supply workstations in EMUs bases. The old material warehouses have been completely merged into a unitary central warehouse in the base. The supply and settlement of materials are also in charge by the supply workstation. The allocation of materials are calculated by material administrators, who will go to be finished in limited time. Variety means of transport can be used, but collaborative operation is difficult, etc [5].

There are two main steps in materializing the logistic process: first enable users to make and print out delivery plans according to their requirements, and ensure transfer and settlement by signing hard copies (see fig 1); then enable users to $\log$ on the EMUs information system with their cards to sign off the materials, and feedback the result and status on the system (see fig 2).

Mathematically, linear planning solves optimization problems when both object function and limiting conditions are linear. As object functions are linear, their extremes will be optimal points. Linear object functions indicate that the optimal solution exists only in the edge of the feasible regions [6]. Linear planning could be applied in calculating optimal route and staff allocation in delivery according to the different locations of materials in the warehouses and workstations in EMU bases, and allocate staff to fetch materials from different warehouses and deliver them to target work station upon maintenance or repair requests. This process could be divided

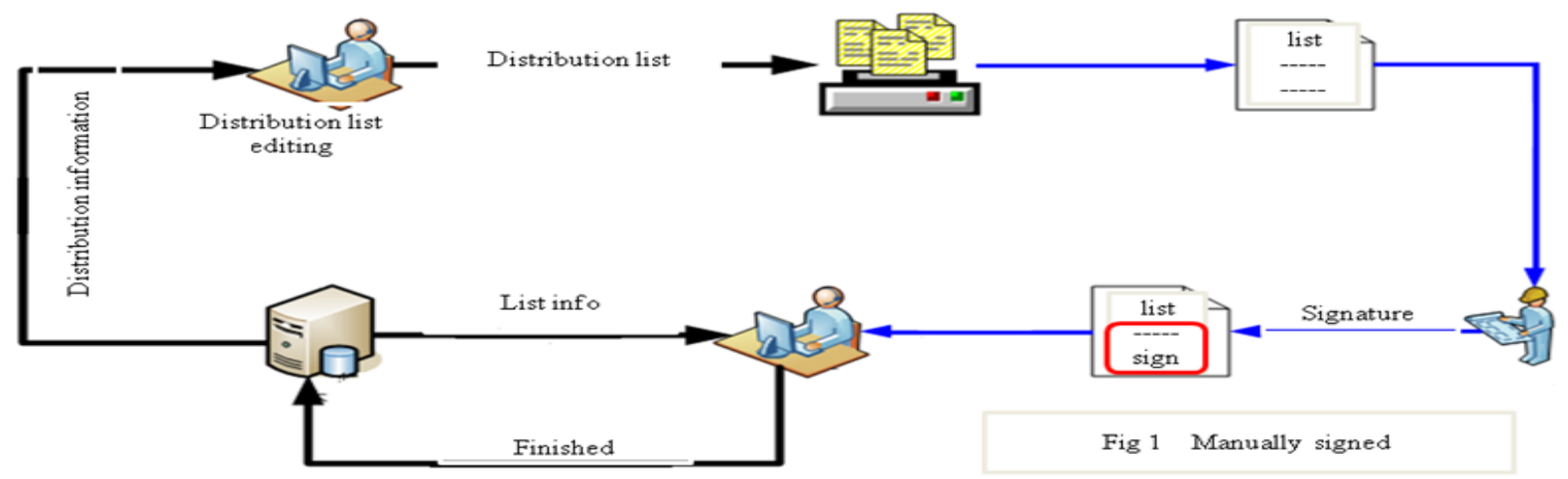

warehouse keepers to fetch the materials to the workstation and notify the users of materials to get them back to their work station, or take the users with them to the warehouse and let them take the material directly to the post.

The delivery principles are as follows: Forming delivery plans based on material applications; forming delivery plans based on maintenance plans; timely delivery to target post on demand; finishing material settlement on post; using both hard and soft copies for settlement.

Several main factors of logistics distribution are considered. The area of production site is too large, and the distance between two work stations is longer. There are many different kinds of parts, also the quantity is huge. The material demand of work station is uncertainty. More maintenance tasks must into car distribution and workstation distribution [7].

The picture below shows the location map of warehouses and workstations in a EMU base. L1 and L3 are material warehouses. Wheel sets are only stored in L3. L2, L4, L5 and L6 are general repair work station, and L7 is a wheel set repair post. In this case both L6 and L7 need materials, and according to DijStar algorithm, the optimal route is L1-L3L2-L5-L6-L7=19. Assume delivery to every station needs 3 minutes, then if $\mathrm{L} 7$ wants to get the material in 10 minutes, the optimal route would be L1-L3-L6-L7 =20. (See fig 3)

Of course, optimal solutions could not always be found, and there are two such scenarios in linear planning. The first is when certain limiting conditions are contradictory, which

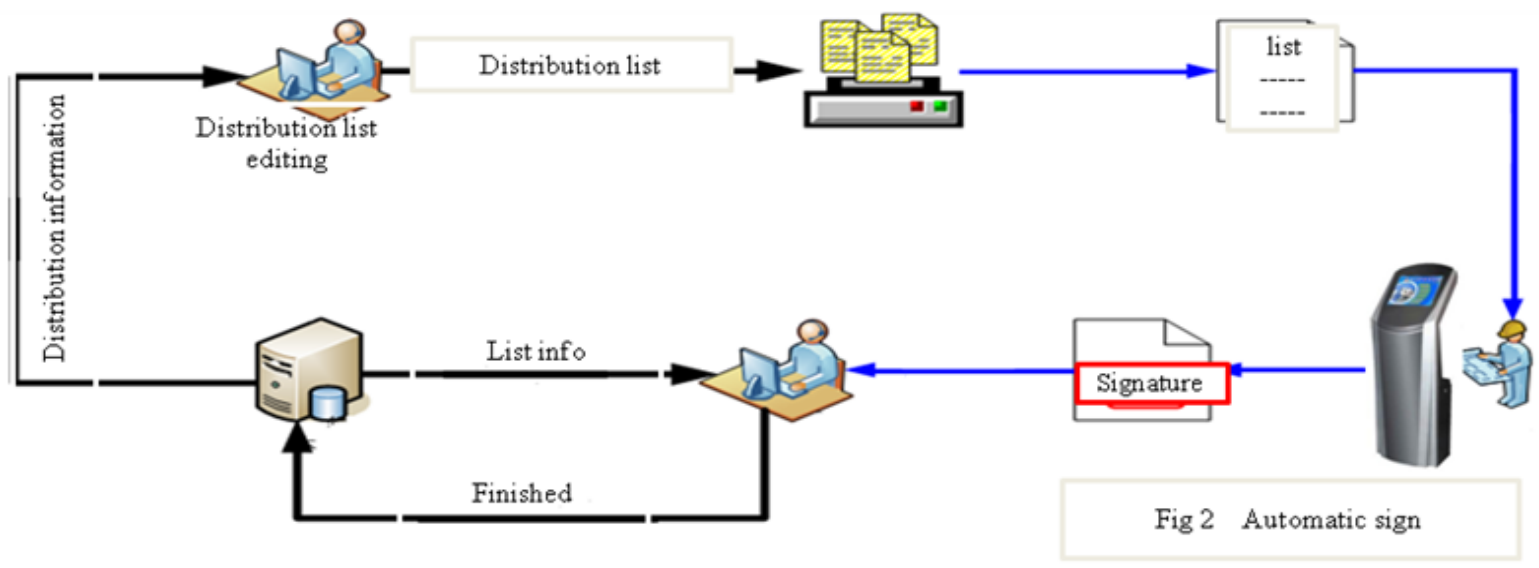


makes the feasible region invalid, and hence no solutions could be found. The other is when the polyhedral of limiting conditions are unbounded on the direction of the object function, which means that the object function could stretch to an arbitrarily large point, and no optimal solutions could be found. Also there could be multiple optimal solutions, covering one edge, line or the whole of a polyhedral.

\section{CONCLUSIONS}

Material delivery in EMUs maintenance in a type of indoor post allocation, in which delivery environment and routes are stable, but frequency and punctuality of delivery are highly important. Detailed management demands that no stock in station or repair units, so it is useful to study how to efficiently allocate material delivery based on production \& maintenance plans, malfunctions and material demands. With linear planning theory in operations research, it is possible to

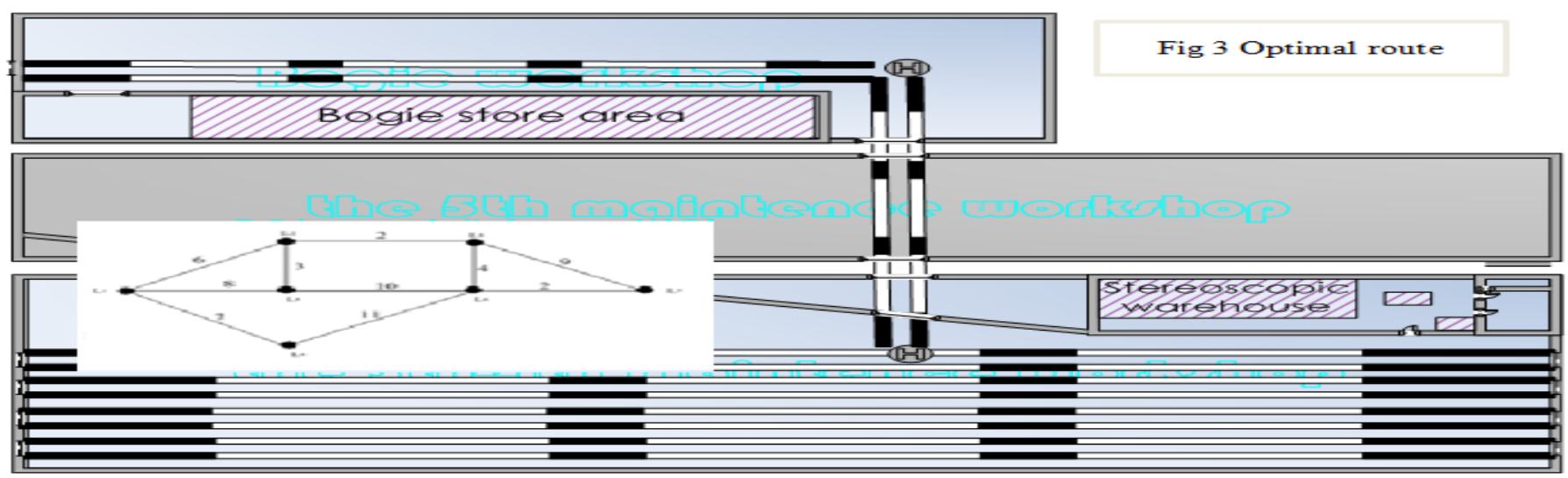

\section{Application OF RFID TeChNOLOGY}

The accuracy and timeliness of the information of maintenance parts is a crucial factor for EMUs logistics. By attaching RFID tags to the key maintenance parts, identification terminals can collect and analyze the information during each stage of the circulation of parts, which can be fully support the maintenance timely and assist the operation of part management efficiently. information was collected conveniently by using the RFID technology.

At the stage of part stockin, the application of the tags to parts at logistic centers makes sure that key parts have the unified logos and unique identifications. It can timely and accurately manage the information, status and direction of each part, which ensures the feasibility of quality tracing and the security of producing, inspecting and operating EMUs. Therefore, the fine management of the key parts can be realized.

At the stage of delivering parts out of storage, the information collection of the key parts can be quickly done through the reading the data at the electronic labels by readerwriters and the interaction with management information system of EMUs. It reduces the manual intervene and improves the working efficiency and data accuracy.

Operators get the only ID of key parts by scanning the RFID tag during the whole maintenance process. Associated with the emu resume database, they can grasp all quality situation of key parts, operation process etc. Also, they will analysis this information to make an effective maintenance conclusion. calculate and design optimal routes for delivery based on different maintenance scenarios. At the same time, process information was collected conveniently by using the RFID technology.

\section{References}

[1] Jiang Zhikai, Introduction to Operations Research Teaching [J], Journal of Chongqing University of Science and Technology (social sciences edition), 2010.

[2] Qian Disong, Operations Research [M], Tsinghua University Press, 1990.

[3] Shen Jiahua., Modern Logistics Operational Research [M], Publication House of Electronic Industry, 2004.

[4] DAI Yuhong, Advances in Linear and Nonlinear Programming, Operations Research Transactions, 2014.

[5] Li Wenquan, the informatization of the logistics distribution system for maintenance base, Shanghai Jiao Tong University for Master Degree of Engineering, 2013.

[6] Wu Xueqin, The Establishment and Application of Linear Programming Model In theTransport of Logistics[J],Journal of Jiangxi Vocationaland Technical College of Electricity,2007.

[7] Equipment Department of Railways Ministry Transportation Management Bureau,EMUs Maintenance Operation Standard,China Railway Publishing House, 2007. 